\title{
ARTICLE OPEN \\ ASC and NLRP3 maintain innate immune homeostasis in the airway through an inflammasome-independent mechanism
}

\author{
Rendong Fang ${ }^{1,2}$, Ryosuke Uchiyama ${ }^{3,7}$, Shunsuke Sakai ${ }^{2,8}$, Hideki Hara ${ }^{2,9}$, Hiroko Tsutsui ${ }^{3}$, Takashi Suda ${ }^{4}$, Masao Mitsuyama ${ }^{2,5}$, \\ Ikuo Kawamura ${ }^{2}$ and Kohsuke Tsuchiya ${ }^{2,4,6}$
}

It is widely accepted that inflammasomes protect the host from microbial pathogens by inducing inflammatory responses through caspase-1 activation. Here, we show that the inflammasome components ASC and NLRP3 are required for resistance to pneumococcal pneumonia, whereas caspase- 1 and caspase-11 are dispensable. In the lung of S. pneumoniae-infected mice, ASC and NLRP3, but not caspase-1/11, were required for optimal expression of several mucosal innate immune proteins. Among them, TFF2 and intelectin-1 appeared to be protective against pneumococcal pneumonia. During infection, ASC and NLRP3 maintained the expression of the transcription factor SPDEF, which can facilitate the expression of the mucosal defense factor genes. Moreover, activation of STAT6, a key regulator of Spdef expression, depended on ASC and NLRP3. Overexpression of these inflammasome proteins sustained STAT6 phosphorylation induced by type 2 cytokines. Collectively, this study suggests that ASC and NLRP3 promote airway mucosal innate immunity by an inflammasome-independent mechanism involving the STAT6-SPDEF pathway.

Mucosal Immunology (2019) 12:1092-1103; https://doi.org/10.1038/s41385-019-0181-1

\section{INTRODUCTION}

The airway surface is continuously exposed to microbes and foreign particles that are inhaled into the respiratory tract. ${ }^{1}$ The airway epithelium forms a protective mucosal barrier between the external and internal environments. Airway epithelial cells, including ciliated and secretory cells, play a key role in the removal of inhaled microbes and particles through mucociliary clearance. Type II alveolar epithelial cells secrete surfactant proteins (SPs) that have protective effects against bacterial pathogens. Hence, the airway epithelium is usually active to maintain an effective mucosal barrier, thereby protecting the host from microbial colonization and infections.

The Gram-positive bacterial pathogen Streptococcus pneumoniae is a leading cause of pneumonia, meningitis, and septicemia and is responsible for significant mortality and morbidity worldwide. $^{2}$ The polysaccharide capsule and the cytolysin pneumolysin (PLY) are critical virulence factors of S. pneumoniae. PLY is a member of cholesterol-dependent cytolysins and forms ring- or arc-shaped pores on cholesterol-containing membranes. PLY plays a critical role in the pathogenicity of $S$. pneumoniae by disrupting tissue barriers, suppressing ciliary beating on epithelial cells, and inhibiting bactericidal activity of neutrophils. ${ }^{3,4}$

Pattern-recognition receptors (PRRs), such as Toll-like receptors (TLRs) and Nod-like receptors (NLRs), play a central role in innate immunity. ${ }^{5}$ PRRs sense specific pathogen-derived molecular structures and, in turn, provide signals that activate innate and acquired immunity. NLR family, pyrin domain-containing 3 (NLRP3, also known as Cryopyrin) and absent in melanoma 2 (AIM2) are cytoplasmic PRRs, which sense potassium efflux and cytosolic DNA, respectively. ${ }^{6,7}$ After recognition of specific agonizts, NLRP3 and AIM2 form inflammasomes. Inflammasomes are cytoplasmic multiprotein complexes that recruit and activate caspase- 1 , which in turn processes pro-interleukin (IL)-1 $\beta$ and pro-IL-18 into their respective bioactive forms. Apoptosis-associated speck-like protein containing a CARD (ASC, also known as PYCARD) is an inflammasome adapter protein that is required for the formation of the AIM2 and NLRP3 inflammasomes. After inflammasome activation, ASC also forms the ASC speck, a supramolecular aggregate of ASC dimers, which serves as another platform for the activation of caspase-1.

Pneumococcal components and products are sensed by several PRRs, leading to the induction of inflammatory mediators, some of which contribute to host defense against $S$. pneumoniae. ${ }^{8}$ Previous studies have suggested that $S$. pneumoniae induces the activation of caspase- 1 and secretion of bioactive IL-1 $\beta$ and IL-18 in a PLYdependent manner and that the AIM2 and NLRP3 inflammasomes mediate the S. pneumoniae-induced activation of caspase- 1 and secretion of these cytokines. ${ }^{8-12}$

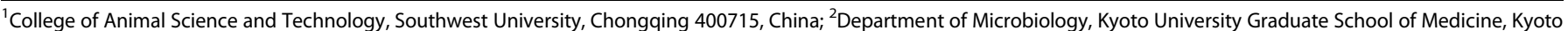

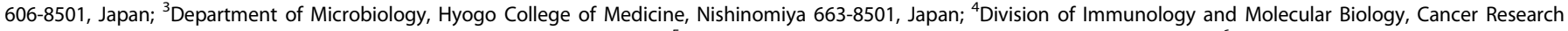

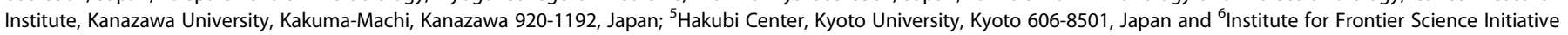
(InFiniti), Kanazawa University, Kanazawa 920-1192, Japan

Correspondence: Kohsuke Tsuchiya (ktsuchiya@staff.kanazawa-u.ac.jp)

${ }^{7}$ Present address: School of Pharmacy and Pharmaceutical Sciences, Mukogawa Women's University, Nishinomiya 663-8179, Japan

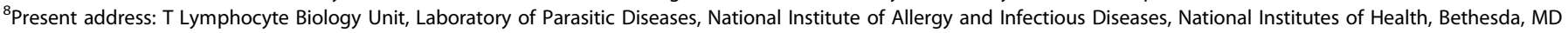
20892, USA

${ }^{9}$ Present address: Department of Pathology and Comprehensive Cancer Center, University of Michigan Medical School, Ann Arbor, MI 48109, USA

These authors contributed equally to this study: R. Fang, R. Uchiyama, S. Sakai
}

Received: 4 July 2018 Revised: 29 May 2019 Accepted: 6 June 2019

Published online: 5 July 2019 
a

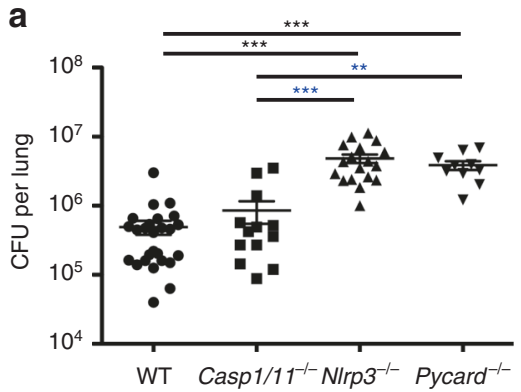

b

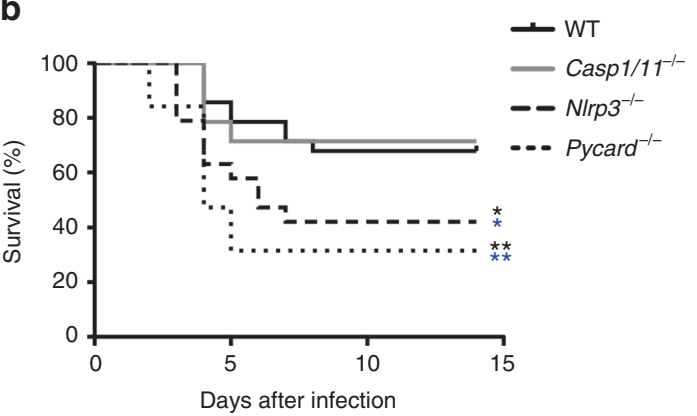

C
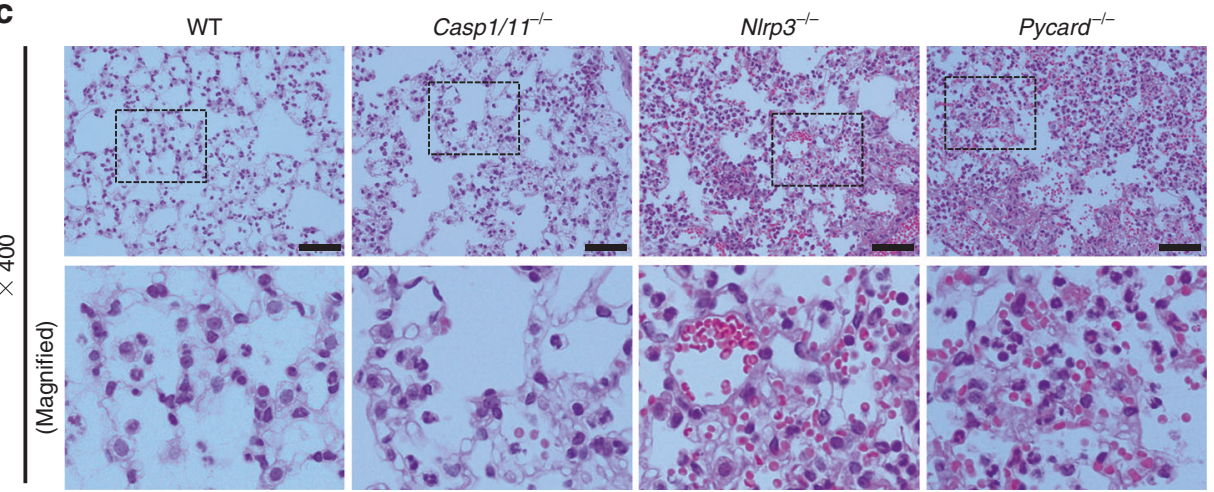

Fig. 1 Caspase-1 is dispensable for host defense against S. pneumoniae. a CFU counts in the lung of the indicated mice collected at $48 \mathrm{~h}$ after intranasal infection with $5 \times 10^{7} \mathrm{CFU}$ of S. pneumoniae. Bars indicate mean \pm SEM. ${ }^{* *} p<0.01 ;^{* * *} p<0.001$ by the Kruskal-Wallis test followed by Dunn's test. Black asterisks represent comparison with WT mice, and blue with Casp $1 / 11^{-1-}$ mice. b Survival curves of the indicated mice infected with S. pneumoniae and monitored for 2 weeks $\left(n=28,28,19\right.$, and 19 for WT, Casp $1 / 11^{-1-}$, Nlrp $3^{-1-}$, and Pycard ${ }^{-1-}$ mice, respectively). ${ }^{*} p<0.05 ;{ }^{* *} p<0.01$ by the log-rank test. Black asterisks represent comparison with WT mice, and blue with Casp $1 / 11^{-1-}$ mice. c H\&E staining images of lung sections of the indicated mice infected with $S$. pneumoniae for $48 \mathrm{~h}$ ( $n=3$ per group). Scale bars, $50 \mu \mathrm{m}$

Except for one study which demonstrated that ASC and NLRP3 impair host defense against serotype 3 S. pneumoniae, ${ }^{13}$ most of previous works have shown that mice deficient in either NLRP3 or ASC were more susceptible to pneumococcal pneumonia than wild-type (WT) mice, suggesting the importance of the NLRP3 inflammasome in host defense against $S$. pneumoniae..$^{9,10,12}$ Moreover, IL-1 $\beta$ and IL-18 have been suggested to play a protective role in pneumococcal pneumonia. ${ }^{14-16}$ In light of the previous observations, one could propose a simple model, in which caspase- 1 contributes to host defense in pneumococcal pneumonia through the processing of IL-1 $\beta$ and IL-18 following NLRP3 inflammasome activation. However, our recent experiments demonstrated that the resistance of caspase-1/caspase-11deficient mice to pneumococcal pneumonia was comparable to that of WT mice. Besides, although NLRP3 is required for optimal host defense, substantial levels of IL- $1 \beta$ and IL- 18 were detected in bronchoalveolar lavage fluid (BALF) of NLRP3-deficient mice infected with S. pneumoniae. Accordingly, NLRP3 and ASC might protect the host from $S$. pneumoniae by a yet unidentified mechanism, which is independent of caspase-1. In this study, we tried to elucidate the mechanism, by which NLRP3 and ASC contribute to the control of pneumococcal pneumonia in an inflammasome-independent manner.

\section{RESULTS}

NLRP3 and ASC, but not caspase-1/11, are required for the resistance against $S$. pneumoniae

To examine the role of inflammasome proteins in pneumococcal pneumonia, WT mice and mice deficient in caspase-1, ASC, or NLRP3 were infected intranasally with $S$. pneumoniae. As previously reported, ASC-deficient $\left(\right.$ Pycard $\left.^{-1-}\right)$ and NIrp3 $^{-1-}$ mice showed higher bacterial burdens in the lung and lower survival rates than WT mice after S. pneumoniae infection (Fig. 1a, b). ${ }^{9,10,12}$ However, there was no significant difference between WT and caspase-1/11-deficient (Casp1/11 ${ }^{-1-}$ ) mice in survival and bacterial counts in the lung. Moreover, severe tissue damage was observed in the lung of Pycard $^{-1-}$ or NIrp3 $3^{-1-}$ mice after S. pneumoniae infection, while the pathogen caused less lung tissue damage in WT or Casp $1 / 11^{-1-}$ mice (Fig. 1c). Many red blood cells were seen in the alveolar spaces of Pycard ${ }^{-1-}$ or Nlrp3 $3^{-1-}$ mice, but not or less in those of WT or Casp $1 / 11^{-1-}$ mice. These results suggest that ASC and NLRP3 protect the host from pneumococcal pneumonia in an inflammasome-independent manner.

Cytokines and neutrophil infiltration do not explain antipneumococcal defense mediated by NLRP3

To elucidate the mechanism, by which ASC and NLRP3 mediate host defense against pneumococcal pneumonia, we examined the role of these inflammasome proteins in innate immune responses to $S$. pneumoniae, such as cytokine production and neutrophil recruitment to the lung. As previously described, BALF levels of IL$1 \beta$ and IL-18 were significantly lower in Pycard $^{-\prime-}$ mice than in WT mice after S. pneumoniae infection (Fig. 2a, b). ${ }^{9}$ However, as low, but measurable, levels of these cytokines were detected in BALF of Pycard ${ }^{-1-}$ mice, it is premature to conclude that IL-1 and IL-18 signals were abolished in Pycard ${ }^{-1-}$ mice. Besides, IL-1a, which signals through the same receptor as IL- $1 \beta$, was produced during S. pneumoniae infection even in the absence of ASC, NLRP3, or caspase-1/11 (Fig. 2c). Substantial levels of IL-1 $\beta$ and IL-18 were detected in BALF of $\mathrm{NIrp3}^{-1-}$ mice (Fig. 2a, b); the protective action of NLRP3 is unlikely to involve the production of these cytokines. In Casp $1 / 11^{-1-}$ mice, although the production of IL-18 in response to $S$. pneumoniae was diminished, that of IL-1 $\beta$ was normal (Fig. 2a, b). The levels of IL-6, TNFa, IL-10, and IFN- $\gamma$ in BALF were substantially increased after $S$. pneumoniae infection in all 


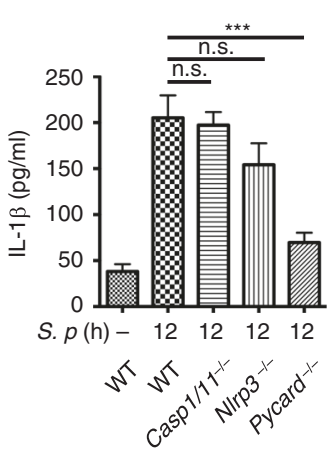

e

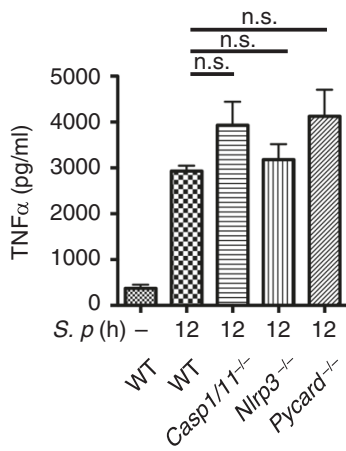

i

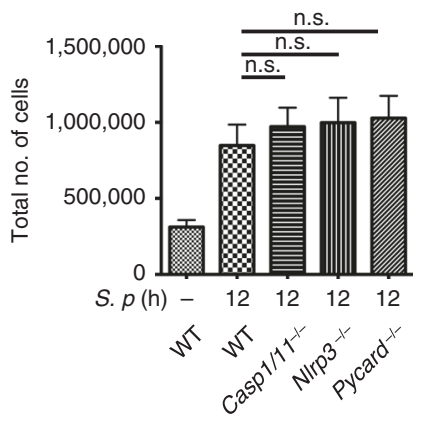

b

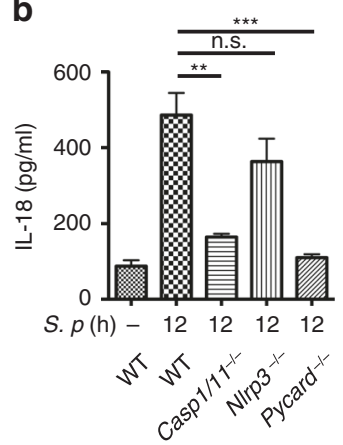

f

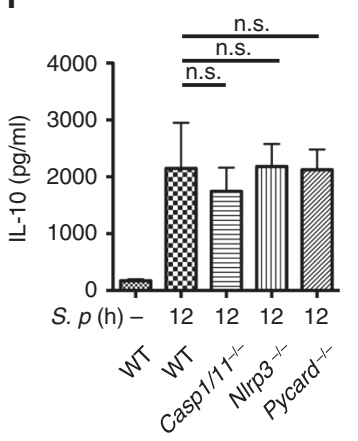

C

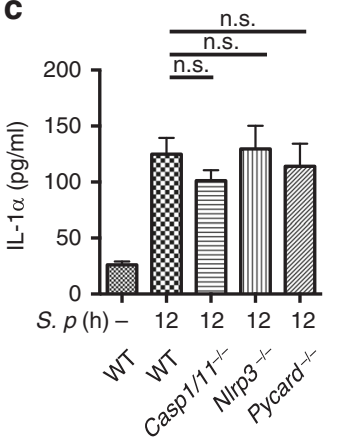

g

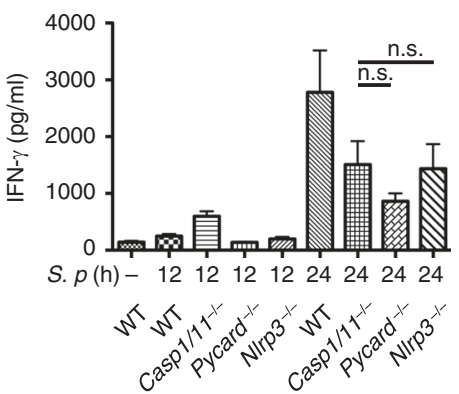

$\mathbf{k}$
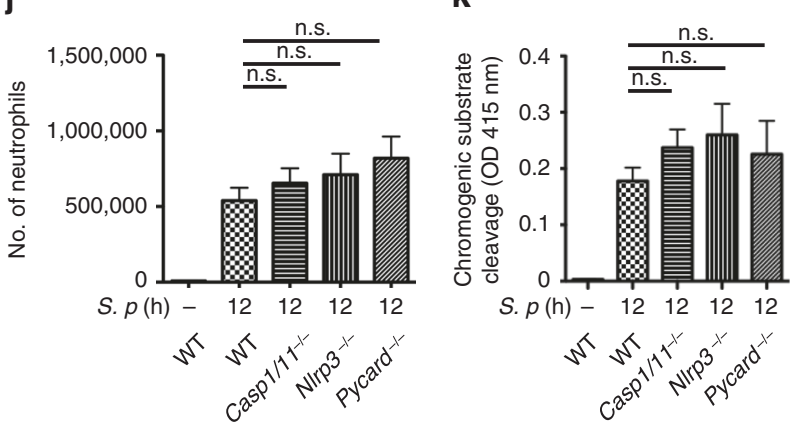

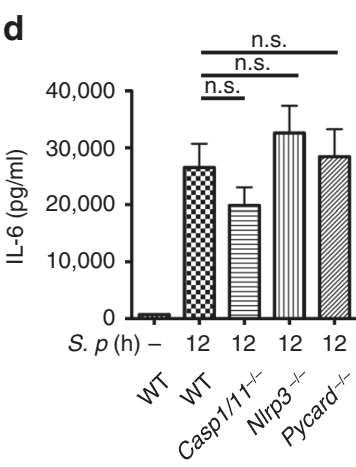

h

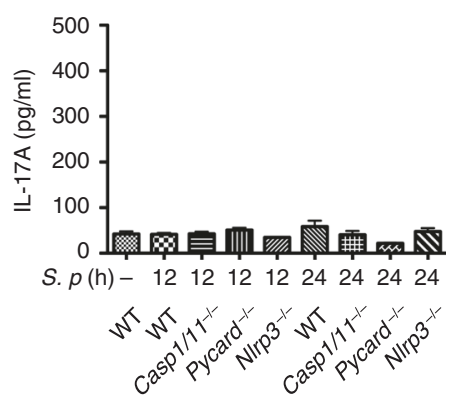

I

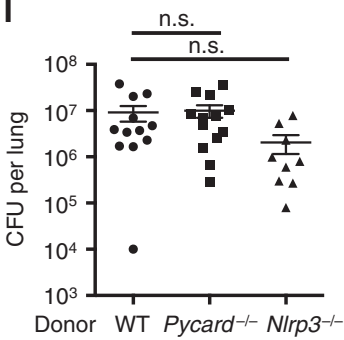

Donor WT Pycard--- NIrp3-/-

Fig. 2 ASC and NLRP3 protect the host through an unknown mechanism. a-g Quantification of IL-1 $\beta$ (a), IL-18 (b), IL-1 $\alpha$ (c), IL-6 (d), TNF $\alpha$ (e), IL-10 (f), IFN- $\gamma(\mathbf{g})$, and IL-17A (h) by ELISA in BALF of the indicated mice infected intranasally for 12 or $24 \mathrm{~h}$ or not with $5 \times 10^{7}$ CFU of S. pneumoniae ( $n=6-19)$. $\mathbf{i}, \mathbf{j}$ Quantification of total number of cells (i) and the number of neutrophils (j) in BALF of the indicated mice infected for $12 \mathrm{~h}$ or not with S. pneumoniae $(n=6-11)$. $\mathbf{k}$ NSP activity in BALF was assessed with a chromogenic substrate $(n=6-8)$. I Lethally irradiated WT mice were reconstituted with bone marrow cells from the indicated donor mice. The bone marrow chimeras were infected as (a), and CFU counts in the lung were determined $48 \mathrm{~h}$ after infection. Data are presented as the mean and SEM. ${ }^{*} p<0.05 ;{ }^{* *} p<0.01 ;{ }^{* * *} p<0.001 ;$ n.s. not significant by the Kruskal-Wallis test followed by Dunn's test (a-I)

groups tested (Fig. 2d-g). IL-17A was not significantly increased in BALF after infection with S. pneumoniae (Fig. 2h). In addition, the number of total cells, neutrophils, and macrophages in BALF were not significantly different among the four groups after $S$. pneumoniae infection (Fig. $2 \mathrm{i}$, j; data not shown). Neutrophilderived serine proteases (NSPs) have been proposed to kill $S$. pneumoniae. The activity of NSPs in BALF samples was increased after infection with $S$. pneumoniae; there was no significant difference in the activity among WT, Casp $1 / 11^{-1-}, \mathrm{NIrp3^{-/- }}$ and Pycard ${ }^{-1-}$ mice (Fig. 2k). Hence, it is suggested that NLRP3 and ASC are dispensable for the recruitment of neutrophils and release of NSPs. Furthermore, we tested whether ASC and NLRP3 in cells of hemopoietic origin is involved in host defense against pneumococcal pneumonia using irradiated bone marrow chimeric mice. There was no significant difference in bacterial counts in the lung between WT recipient mice reconstituted with bone marrow cells from WT mice and those from Pycard ${ }^{-1}$ mice or Nlrp3 ${ }^{-1-}$ mice (Fig. 2l; Fig. S1). From these results, we speculate that NLRP3 and
ASC protect the host from S. pneumoniae through mechanisms other than inflammasome-dependent cytokine production by immune cells.

ASC- and NLRP3-dependent gene expression in the lung To know how ASC and NLRP3 protect the host from $S$. pneumoniae, we attempted to identify genes regulated by ASC and NLRP3 and performed a microarray analysis using total RNAs extracted from whole lungs of WT and mutant mice that had been infected with S. pneumoniae. The microarray analysis revealed 213 genes and non-coding RNAs (there are overlapping) that were downregulated by deficiency of ASC or NLRP3, but not by that of caspase-1/11 (Fig. 3a; Table S1). Several of these genes encode mucosal innate immune proteins, such as regenerating isletderived $3 \gamma($ REG3 $\gamma$ ), trefoil factor 2 (TFF2), BPI fold containing family A member 1 (BPIFA1), BPIFB1, intelectin-1, and mucin 5b (Fig. 3a). ${ }^{17-25}$ On the other hand, the expression of genes coding for other markers of pulmonary epithelial cells (SCGB1A1, SP-A, 
a

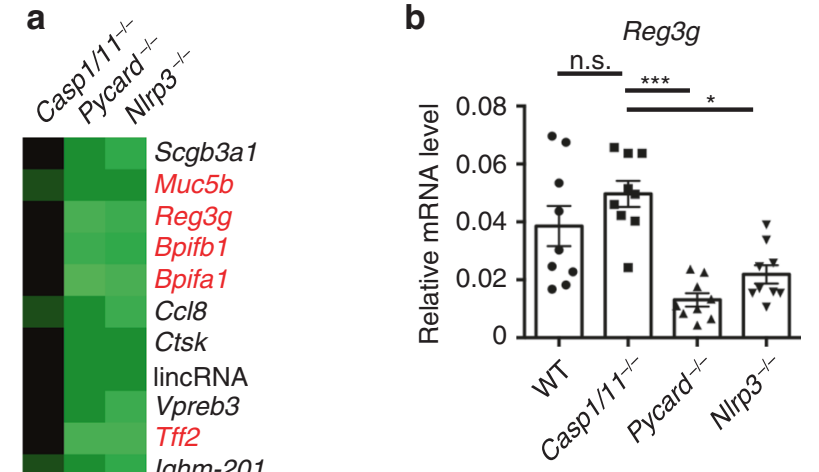

C

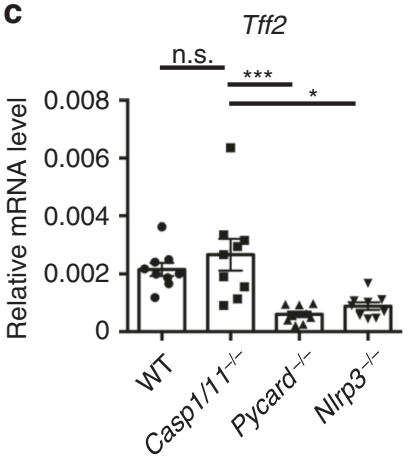

e

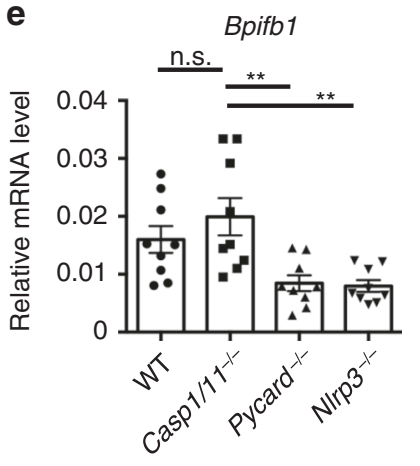

f
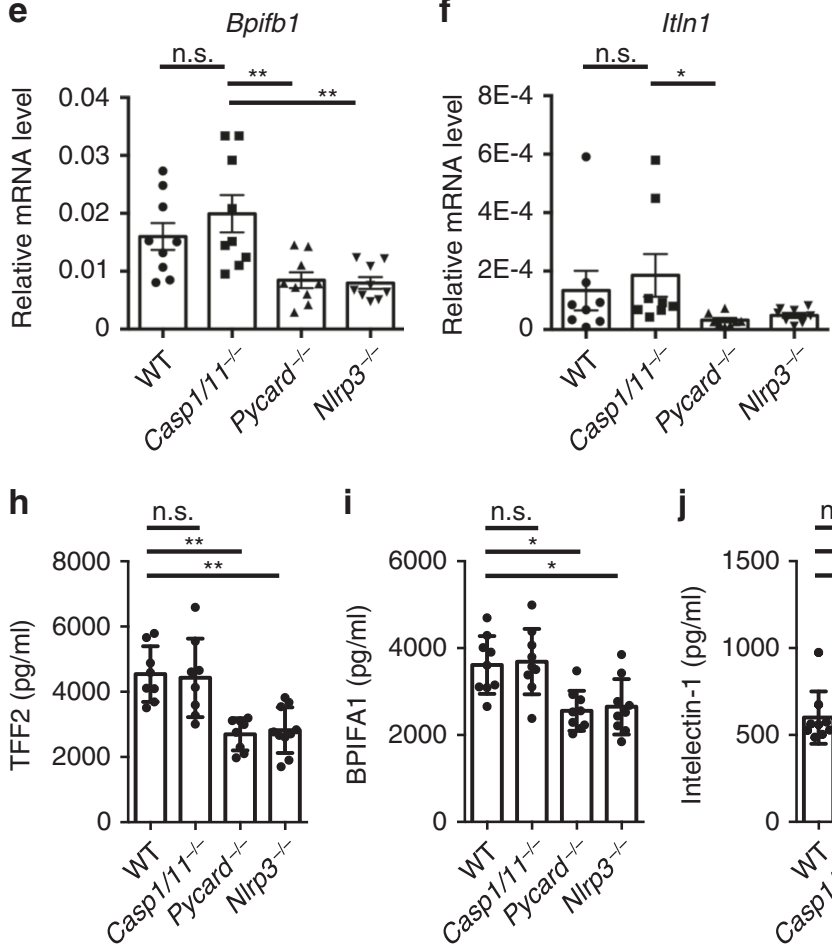

i

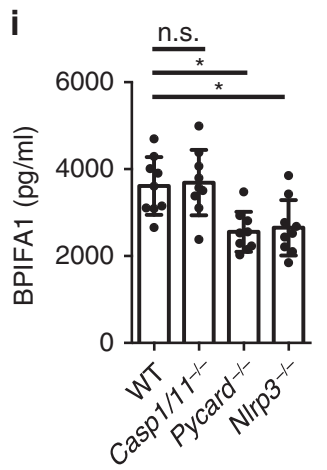

4930451C15Rik

2610507101Rik

Speer5-ps1

Log2 ratio to WT

$\begin{array}{llllll}-3 & -2 & -1 & 1 & 2 & 3\end{array}$

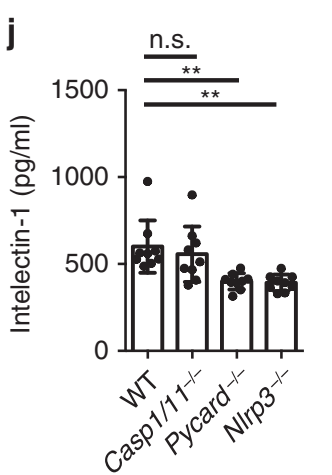

d

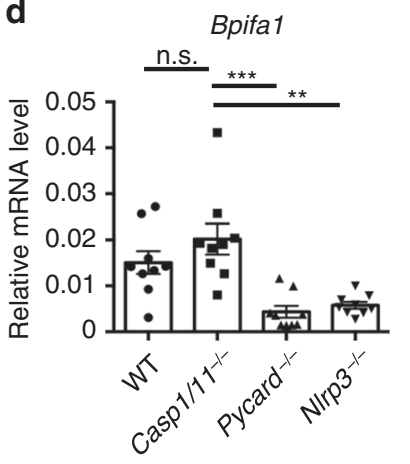

g

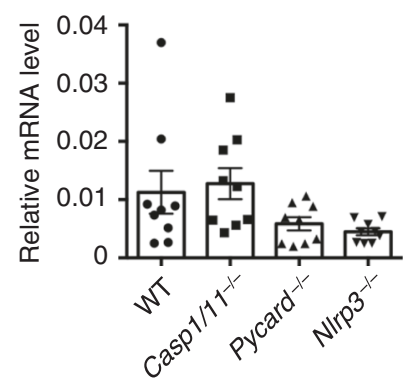

Fig. 3 ASC and NLRP3 regulate the expression of mucosal defense factors. a Gene-expression profiling by microarray analysis in the lung of the indicated mice infected intranasally with $5 \times 10^{7}$ CFU of S. pneumoniae for $24 \mathrm{~h}$. Total RNA samples from two mice in each group were mixed before analysis. Data are expressed as log 2 ratio to WT mice. Genes with a log 2 ratio less than -1.2 and larger than -0.7 are considered downregulated and not downregulated, respectively. The top 30 genes in signal intensity among the genes downregulated by deficiency of ASC and NLRP3 (log2 ratio $<-1.2$ ), but not by that of caspase-1 (log2 ratio $<-0.7$ ), are shown. $\mathbf{b}-\mathbf{g}$ Quantitative RT-PCR for assessing the levels of Reg3g (b), Tff2 (c), Bpifa1 (d), Bpifb1 (e), Itnl1 (f), and Muc5b (g) mRNA relative to Actb mRNA in the lung of the indicated mice $24 \mathrm{~h}$ after infection as a ( $n=9$ in each group). Data are presented as the mean and SEM. h-k Levels of TFF2 (h), BPIFA1 (i), intelectin-1 (j), and MUC5B (k) assessed by ELISA in lung homogenates of the indicated mice $24 \mathrm{~h}$ after infection as (a) $(n=8-10$ in each group). Data are presented as the mean \pm SD. ${ }^{*} p<0.05 ;{ }^{* *} p<0.01 ;{ }^{* * *} p<0.001 ;$ n.s. not significant by the Kruskal-Wallis test followed by Dunn's test

SP-B, SP-C, SP-D, podoplanin, aquaporin-5, E-cadherin, and epithelial cell adhesion molecule) and housekeeping proteins (GAPDH, $\beta$-actin, and tubulins) was not decreased in the absence of caspase-1, ASC, or NLRP3 (Table S2). Moreover, deficiency of NLRP3 or ASC did not reduce the expression of plasminogen activator inhibitor-1 (also known as SERPINE1), which has been reported to prevent severe lung injury after $S$. pneumoniae infection (Table S2). ${ }^{26}$ We confirmed by quantitative RT-PCR that the expression of Reg3g, Tff2, Bpifa1, and Bpifb1 was lower in the lung of Pycard ${ }^{-1-}$ or Nlrp3 $3^{-1-}$ mice than in that of WT or Casp1/ $11^{-1-}$ mice, (Fig. 3b-e). The expression of Muc5 $b$ and It In 1 showed a tendency to be decreased in the absence of ASC or NLRP3 (Fig. $3 f-g)$. Protein levels of TFF2, BPIFA1, intelectin-1, and mucin $5 \mathrm{~b}$ were significantly lower in Pycard ${ }^{-1-}$ and $\mathrm{Nlrp3}^{-1-}$ mice than in WT mice (Fig. 3h-k). Taken together, ASC and NLRP3 appear to regulate the expression of several pulmonary epithelial genes, whose products might be involved in innate immunity and mucosal barrier function, in an inflammasome-independent manner.

Notably, in WT mice, the expression of Reg3g, Tff2, and Bpifa1 was not increased by S. pneumoniae infection; rather, that of Tff2 and Bpifa1 was decreased during infection (Fig. S2). In ASCdeficient mice, expression levels of these genes were significantly lower than those in WT and caspase-1/11-deficient mice both before and during S. pneumoniae infection (Fig. S2). On the other hand, loss of NLRP3 did not affect the expression of Reg $3 g$, Tff2, and Bpifa 1 in the lung of uninfected mice, whereas the expression was more severely reduced in NLRP3-deficient mice than in WT and caspase-1/11-deficient mice during $S$. pneumoniae infection (Fig. S2). These results suggest that ASC maintains the expression of Reg $3 g$, Tff2, and Bpifa1 under normal conditions and that ASC and NLRP3 have an inhibitory effect on the reduction in the 


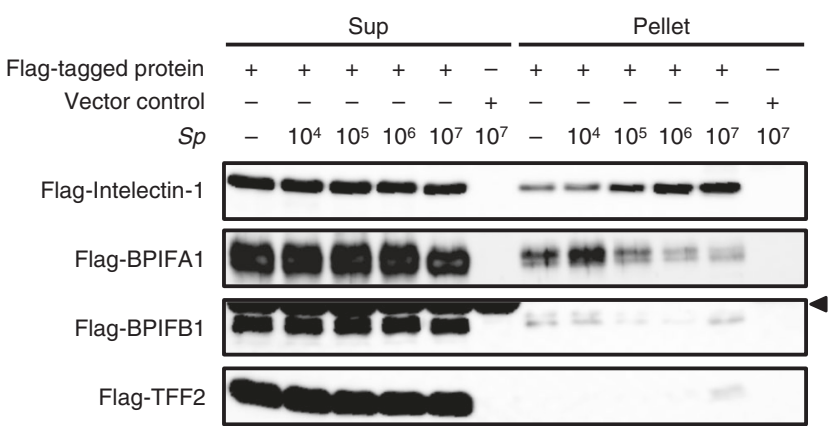

d

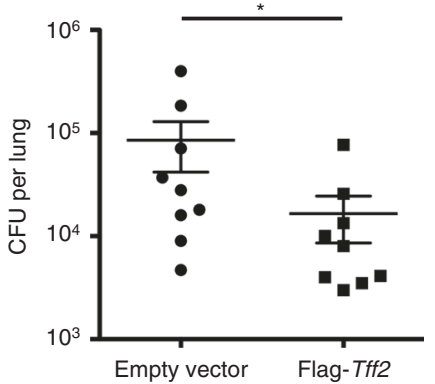

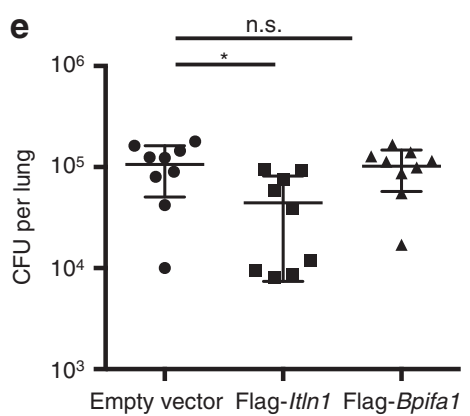

b

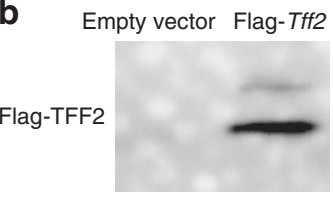

C

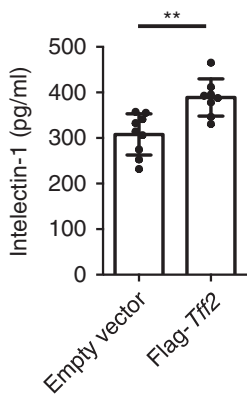

Fig. 4 TFF2 and intelectin-1 protect from pneumococcal pneumonia. a S. pneumoniae pull-down assays for the indicated Flag-tagged proteins. The indicated CFU of S. pneumoniae were incubated at $4{ }^{\circ} \mathrm{C}$ for $2 \mathrm{~h}$ with culture supernatants of HEK 293 cells transfected with each protein. After washing, proteins bound to bacterial cells were eluted with SDS-sample buffer and detected by immunoblotting with an anti-Flag Ab. The experiments were performed three times with similar results. Filled triangle indicates non-specific bands. b Western blot analysis of Flagtagged TFF2 in BALF of Pycard ${ }^{-1-}$ mice transfected intranasally with $10 \mu \mathrm{g}$ of Flag-Tff2 expression vector or empty vector for $24 \mathrm{~h}$. BALF samples were subjected to immunoblotting with an anti-Flag Ab. c Quantification of intelectin-1 in the lung of Pycard ${ }^{-1-}$ mice transfected intranasally with $10 \mu \mathrm{g}$ of Flag-It/n1 expression vector or empty vector for $24 \mathrm{~h}$. Lung homogenates were subjected to ELISA for intelectin-1. Data are presented as the mean \pm SD. $(n=8-9)$. d, e Pycard $^{-1-}$ mice were intranasally transfected with Flag-Tff2 (d), Flag-Itln1 (e), or Flag-Bpifa1 (e) vectors or an empty vector. The mice were infected with $10^{7} \mathrm{CFU}$ of S. pneumoniae $24 \mathrm{~h}$ after transfection, and CFU counts in the lung were determined $48 \mathrm{~h}$ after infection. Bars indicate mean \pm SEM $(n=9) .{ }^{*} p<0.05 ;{ }^{* *} p<0.01 ;$ n.s. not significant by the Kruskal-Wallis test followed by Dunn's test (e) or by the Mann-Whitney $U$ test $(\mathbf{c}, \mathbf{d})$

expression of the mucosal defense genes during $S$. pneumoniae infection. We also found that Reg3g expression in the distal jejunum was significantly lower in the absence of ASC than in its presence (Fig. S3), supporting that ASC is involved in the expression of this gene.

Involvement of TFF2 and intelectin-1 in host defense against pneumococcal pneumonia

It has been reported that REG3y, BPIFA1, and intelectin-1 bind to certain bacteria to kill, inhibit the growth, or assist phagocytosis of them. REG3y has been shown to minimally bind with $S$. pneumoniae. ${ }^{17,20,22,25,27}$ We analyzed the binding ability of BPIFA1, BPIFB1, intelectin-1, and TFF2 to $S$. pneumoniae. Flag-tagged proteins of BPIFA1, BPIFB1, and TFF2 were not pulled-down with $S$. pneumoniae cells (Fig. 4a), suggesting that these proteins hardly bind to pneumococci. On the other hand, intelectin-1 showed binding to $S$. pneumoniae (Fig. 4a), suggesting a possible contribution of this protein to the protection against pneumococcal pneumonia. The epithelial repair factor TFF2 has recently been implicated in host defense against microbial infections. ${ }^{19,28}$ We examined the effect of TFF2, intelectin-1, and BPIFA1 on pneumococcal pneumonia. Pycard ${ }^{-1-}$ mice were transfected intranasally with expression vectors for TFF2, intelectin-1, or BPIFA1 or an empty vector and then infected with S. pneumoniae. Flag-tagged TFF2 was detected in BALF from TFF2-transfected mice, but not in that from empty vector-transfected mice (Fig. 4b). The levels of intelectin-1 were increased in the lung of Pycard ${ }^{-1-}$ mice after transfection with intelectin-1 (Fig. 4c). Strikingly, bacterial counts in the lung of the TFF2 and intelectin- 1 vector groups were lower than those in the lung of the empty vector group (Fig. 4d, e). On the other hand, bacterial counts in the lung of the BPIFA1 vector group showed no difference comparing with those in the empty vector group (Fig. 4e). These results suggest that TFF2 and intelectin-1 are protective against pulmonary pneumococcal infection.

ASC and NLRP3 are dispensable for the activation of STAT3 and MAPKs

Previous studies have suggested STAT3 and MAPK pathways regulate the expression of Reg3g, Bpifa1, Bpifb1, Muc5b, Scgb3a1, and Tff ${ }^{29-31}$ In addition, a study suggested ASC can mediate the activation of STAT3 and activator protein 1 $(A P-1){ }^{32}$ Then, we tested whether ASC and NLRP3 are involved in the activation of STAT3 and MAPK in the lung. Although phosphorylated STAT3 (phospho-STAT3) was detected in airway epithelial cells in both infected and uninfected mice, it was not reduced in the absence of ASC or NLRP3 (Fig. S4A-C). Moreover, phospho-JNK and phospho-extracellular signal-regulated kinases 1/2 (ERK), upstream signaling kinases of AP-1, were not significantly lower in the lung of Pycard ${ }^{-1-}$ mice and $N / r p 3^{-1-}$ mice than in that of WT mice (Fig. S4A, B). Hence, activation of STAT3, JNK, and ERK is unlikely to depend on ASC and NLRP3. The transcriptional regulator NKX2-1 (also known as TTF1) has been presented to have an inhibitory effect on the expression of Reg3g, Tff2, Muc5b, and $I t \ln 1 .{ }^{33}$ Expression levels of Nkx2-1 in the lung were similar in all groups tested (Fig. S4D, $\mathrm{E}$; Table S2), ruling out a role for ASC and NLRP3 in regulating NKX2-1 expression. 
ASC and NLRP3 facilitate STAT6 activation and SPDEF expression Sterile alpha motif pointed domain-containing ets transcription factor (SPDEF), in contrast to NKX2-1, has been shown to positively regulate the expression of Reg $3 g, T f f 2, M u c 5 b$, and $I t \ln 1$ directly or through differentiation of secretory cells (GSE44101)..$^{34-36}$ In HEK293 cells, forced expression of SPDEF increased the promoter activity of the Reg $3 g$ gene, confirming this transcription factor activates the expression of this gene (Fig. 5a). On the other hand, forced expression of ASC and a constitutively active mutant of NLRP3 (NLRP3_R258W) did not increase the promoter activity of the Reg3g gene, suggesting that the NLRP3-ASC pathway has no direct effect on the expression of this gene. We found that the expression of Spdef in the lung was significantly reduced during $S$. pneumoniae infection in Pycard ${ }^{-1-}$ and $\mathrm{Nlrp3}^{-1-}$ mice, whereas the infection did not affect it in WT and Casp $1 / 11^{-/-}$mice (Fig. 5b). These results suggest that ASC and NLRP3 positively regulate the expression of SPDEF during $S$. pneumoniae infection, thereby maintaining the expression of SPDEF-regulated airway defense factors.

Spdef is a STAT6 target gene; for example, IL-13, a type 2 cytokine, has been shown to increase SPDEF expression in a STAT6-dependent manner. ${ }^{37}$ Accordingly, we tested whether STAT6 signaling is activated during $S$. pneumoniae infection. Phospho-STAT6 was increased in the airway epithelium during pneumococcal pneumonia (Fig. 5c; Fig. S5). Notably, the S. pneumoniae induction of phospho-STAT6 was attenuated in Pycard $^{-1-}$ and Nlrp3 $3^{-1-}$ mice. Moreover, the expression of representative STAT6-responsive genes, Muc5ac and Clca3, was significantly lower in the lung of Pycard ${ }^{-1-}$ mice than in that of WT mice. Pulmonary expression of Muc5ac and $\mathrm{Clca} 3$ was also significantly lower in $\mathrm{Nlrp3^{-1- }}$ mice than in WT mice (Fig. $5 \mathrm{~d}$, e). It is therefore suggested that ASC and NLRP3 are involved in STAT6 activation during pneumococcal pneumonia.

\section{ASC and NLRP3 sustain STAT6 phosphorylation}

Type 2 cytokines, including IL-4 and IL-13, induce STAT6 activation. ${ }^{38}$ The expression of IL4 was not detectable by realtime RT-PCR (data not shown). Although the expression of IL13 in the lung of $S$. pneumoniae-infected mice tended to be reduced by deficiency of ASC or NLRP3, IL-13 protein levels in BALF were not significantly different among WT, Casp $1 / 11^{-1-}, \mathrm{Nlrp}^{-/-}$, and Pycard $^{-1-}$ mice (Fig. 6a, b). IL-25, IL-33, and TSLP (thymic stromal lymphopoietin) have been demonstrated to regulate innate type 2 responses. ${ }^{38}$ The expression of these genes in the lung of Nlrp3 ${ }^{-/-}$ and Pycard ${ }^{-1-}$ mice was comparable to that in WT mice (Fig. 6c-e). We also measured the levels of IL-33 in BALF by ELISA, as IL-33 lacks a canonical secretory signal sequence and is released upon necrotic cell death as an alarmin. The levels of IL-33 in BALF of uninfected and infected mice were under the detection limit of the ELISA kit; nonetheless, S. pneumoniae infection seemed to reduce BALF levels of IL-33 in the absence of ASC or NLRP3 (Fig. 6f). However, genetic ablation of IL-1R-like 1 (also known as ST2), a receptor for IL-33, ${ }^{39}$ did not affect the expression of Spdef, Muc5ac, and Tff2 in the lung of S. pneumoniae-infected mice, though Reg3g expression was slightly lower in IL-1R-like 1deficient mice than in WT mice (Fig. $6 \mathrm{~g}-\mathrm{j}$ ). Judging from these results, it is unlikely that IL-33 links ASC and NLRP3 to STAT6 activation and the expression of the mucosal defense genes during pneumococcal pneumonia.

To examine the direct effect of NLRP3 and ASC on STAT6 activation, HEK293 cells transfected with STAT6 were stimulated with recombinant ( $r$ ) IL-13 or IL-4, which has been demonstrated to induce STAT6 phosphorylation in this experimental setting. ${ }^{40}$ Phosphorylated STAT6 was detected in STAT6-transfected HEK293 cells after treatment with rlL-13/4 (Fig. 7a-d; Fig. S6). STAT6 phosphorylation peaked at 1 or $2 \mathrm{~h}$ after rlL-13/4 treatment, while it gradually decreased at later time points, suggesting that STAT6 phosphorylation is suppressed by a negative feedback mechanism during the treatment. Surprisingly, when HEK293 cells were cotransfected with ASC plus NLRP3_R258W, the decrease in STAT6 phosphorylation at the later time points after rlL-13/4 treatment was less pronounced than when the cells were co-transfected with the control vector (Fig. 7a, c; Fig. S6). On the other hand, cotransfection with ASC plus NLRP3_R258W did not sustain STAT6 phosphorylation if the cells were treated with rlL-13 or rlL-4 for $1 \mathrm{~h}$ and then washed to remove the cytokines (Fig. 7b, d; Fig. S6). From these results, it is conceivable that NLRP3 and ASC sustain phosphorylation of STAT6 by inhibiting a negative feedback mechanism, which controls type 2 cytokine induction of STAT6 activation.

\section{DISCUSSION}

It is well-established that caspase- 1 is a central effector of inflammasomes. In fact, a number of studies have suggested that inflammasomes exert their physiological and pathological effects by activating caspase- 1 , which leads to pyroptosis and production of IL-1 $\beta$ and IL-18. ${ }^{6,7}$ In this study, we found that NLRP3 and ASC, but not caspase-1/11, play a critical role in host defense against pneumococcal pneumonia. We also found that during $S$. pneumoniae infection, NLRP3 and ASC maintain the expression of several mucosal defense genes in an inflammasomeindependent manner. Thus, this study provides an insight into innate immune homeostasis in airway mucosa.

A recent report has demonstrated that during $S$. pneumoniae infection, NLRP3 protects the lung epithelial barrier by increasing the adherence of alveolar epithelial cells. ${ }^{41}$ The study also showed that this protective effect of NLRP3 is independent of ASC, caspase- 1 , and IL-1/IL-18. On the other hand, we found that both NLRP3 and ASC are involved in the expression of mucosal defense factors upon pneumococcal pneumonia. Hence, the previous study and our study discovered different inflammasomeindependent roles for NLRP3 in host defense against $S$. pneumoniae, and it is conceivable that NLRP3 protects the host from this pathogen by multiple mechanisms.

A previous study has suggested that IL-18-deficient mice are more susceptible to pneumococcal pneumonia than WT mice. ${ }^{15}$ As most of IL-18 production was caspase-1-dependent during $S$. pneumoniae infection, we had expected that Casp $1 / 11^{-/-}$mice are susceptible to this bacterial pathogen. However, unexpectedly, the resistance of Casp $1 / 11^{-1-}$ mice to S. pneumoniae was comparable to that of WT mice (Fig. 1). A possible explanation for this discrepancy is that IL-18 signaling was not completely abrogated in caspase-1-deficient mice. Indeed, S. pneumoniae-induced IL-18 production was significantly impaired, but not completely abolished, in the absence of caspase-1/11 (Fig. 2b). Substantial levels of IFN- $\gamma$ were also produced in Casp $1 / 11^{-1-}$ mice (Fig. $2 \mathrm{~g}$ ). Therefore, it is conceivable that in Casp $1 / 11^{-/-}$mice, the low levels of IL-18 were produced by a caspase-1-independent mechanism. On the other hand, IL-18 signaling would be completely abrogated in IL-18-deficient mice, which might significantly affect the resistance to pneumococcal pneumonia. It should also be noted that Casp $1 / 11^{-1-}$ mice, but not Casp $1^{-1-}$ mice, were used in this study. Therefore, the possibility that caspase-11 impairs host defense and expression of mucosal defense genes cannot be ruled out and needs further investigations.

TFF2 has been known to play critical roles in mucus gel formation and tissue repair. Although the precise mechanism by which TFF2 contributes to host defense against $S$. pneumoniae remains unclear, this peptide perhaps promotes mucosal barrier function to control pneumococcal pneumonia. It has been reported that TFF2-deficient mice are more susceptible to Yersinia enterocolitica and the hookworm parasite Nippostrongylus brasiliensis, ${ }^{19,28}$ suggesting that TFF2 protects from different types of pathogens. REG3Y and BPIFA1 have also been reported to play 

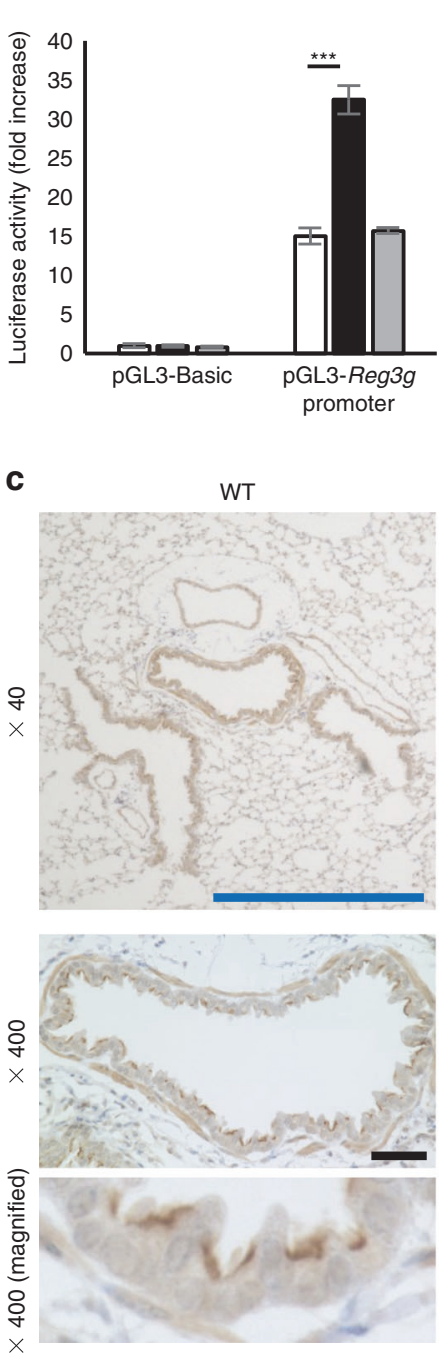

d

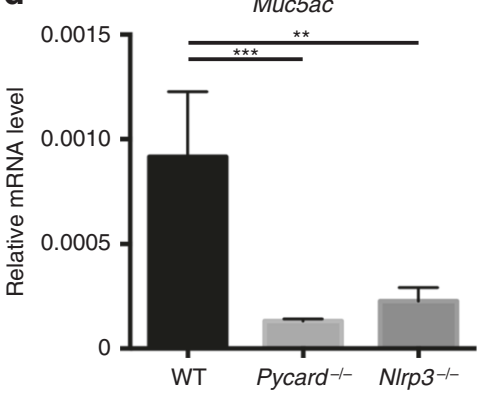

b

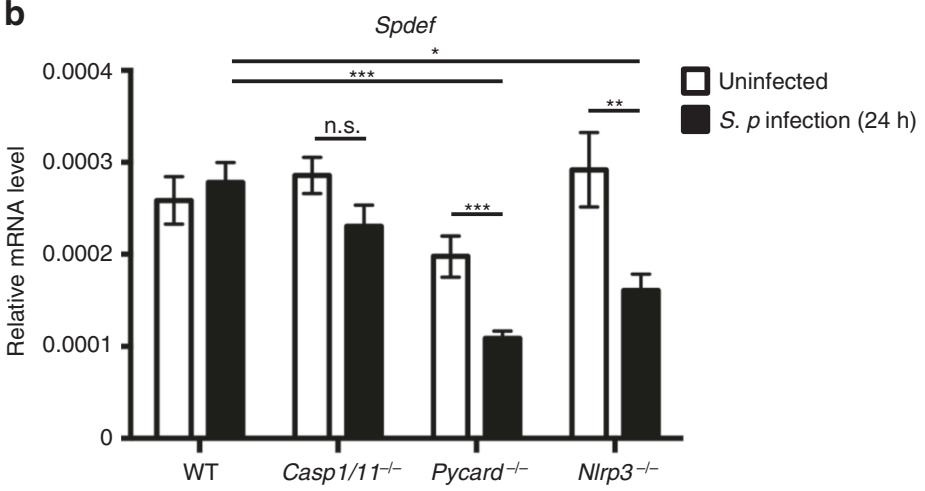

NIrp3-1-
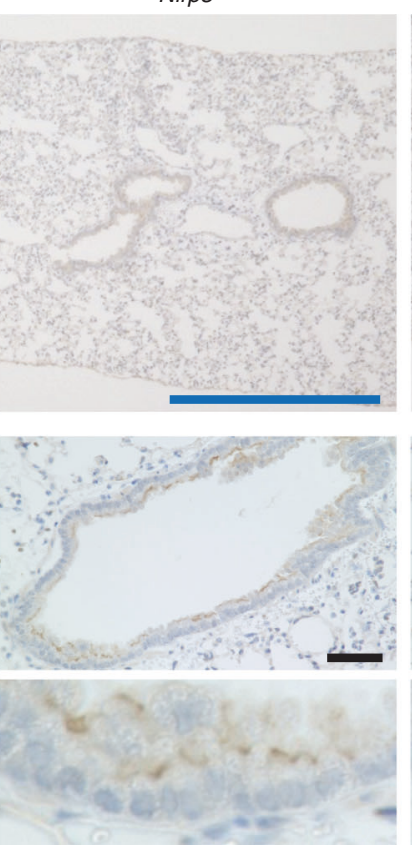

e

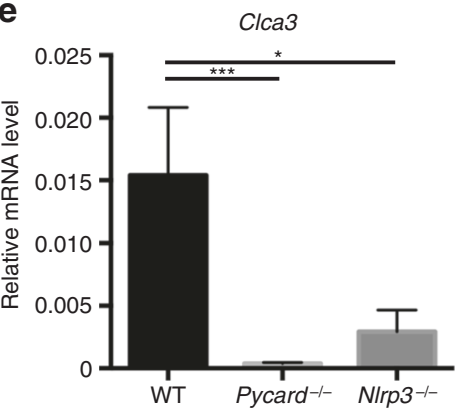

Pycard $^{-1-}$
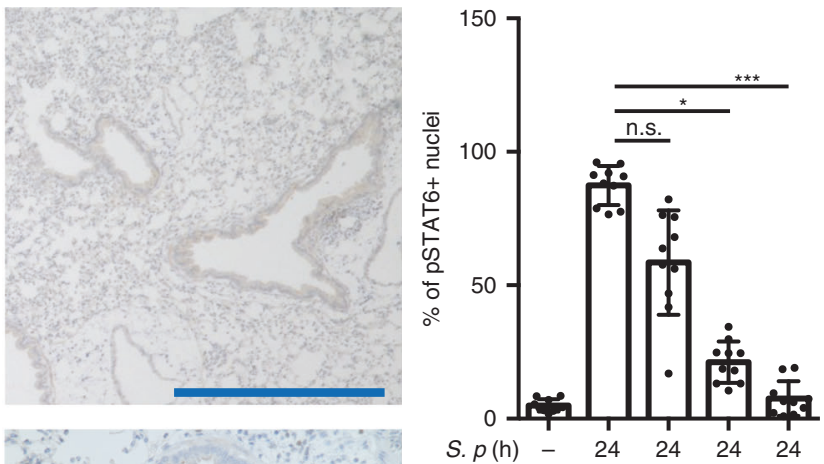

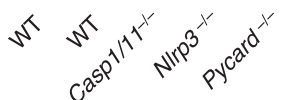

Fig. 5 ASC and NLRP3 maintain the expression of SPDEF. a HEK293 cells were co-transfected with either of the reporter vectors and expression vectors for SPDEF or NLRP3_R258W plus ASC or an empty vector. Luciferase activity in lysates of the cells was measured $48 \mathrm{~h}$ after transfection, and the results are expressed as fold increase in activity over vector control (pGL3-basic/empty expression vector). Data are presented as the mean and SD of triplicate assays. The experiments were repeated three times with similar results. b Quantitative RT-PCR for assessing the levels of Spdef mRNA relative to Actb mRNA in the lung of the indicated mice infected for $24 \mathrm{~h}$ or not with $5 \times 10^{7} \mathrm{CFU}$ of $S$. pneumoniae $(n=9-12)$. c Immunohistochemistry of phospho-STAT6 in lung sections of the indicated mice infected for $24 \mathrm{~h}$ with S. pneumoniae $(n=5)$. Black and blue scale bars represent 50 and $500 \mu \mathrm{m}$, respectively. Right panel shows the percentage of airway epithelial cells, whose nuclei were positive for phospho-STAT6. The data were from 10 airway epithelia in each group (more than 1000 cells per group) and presented as the mean \pm SD. d, e Quantitative RT-PCR for assessing the levels of Muc5AC (d) and Clca3 (e) mRNA relative to Actb mRNA in the lung of the indicated mice infected for $24 \mathrm{~h}$ with S. pneumoniae $(n=9)$. a, d, e Data are presented as the mean and SEM. ${ }^{*} p<0.05 ;{ }^{* *} p<0.01 ;{ }^{* * *} p<0.001$; n.s. not significant by the Kruskal-Wallis test followed by Dunn's test (a-e) or by the Mann-Whitney $U$ test (b) 


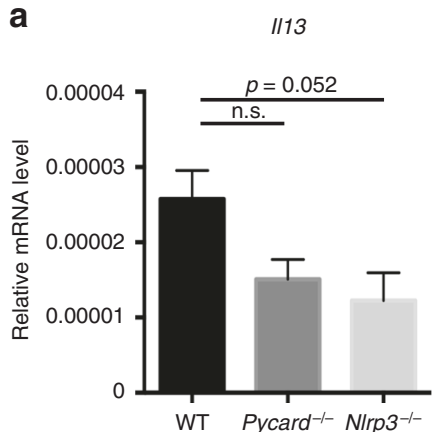

C

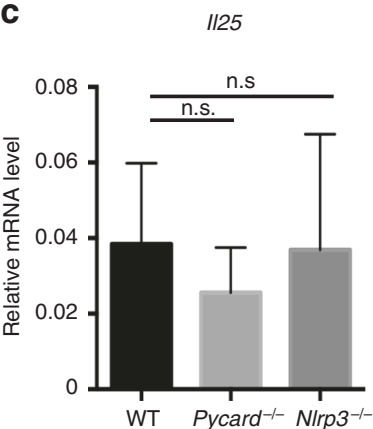

d

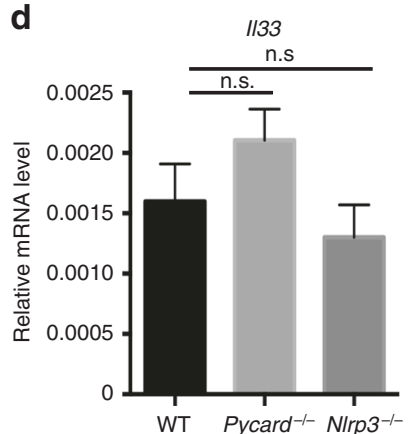

e

Tsip

b

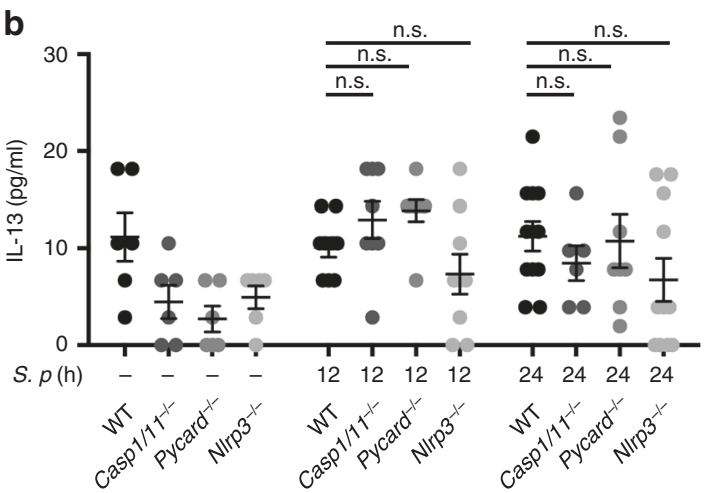

f
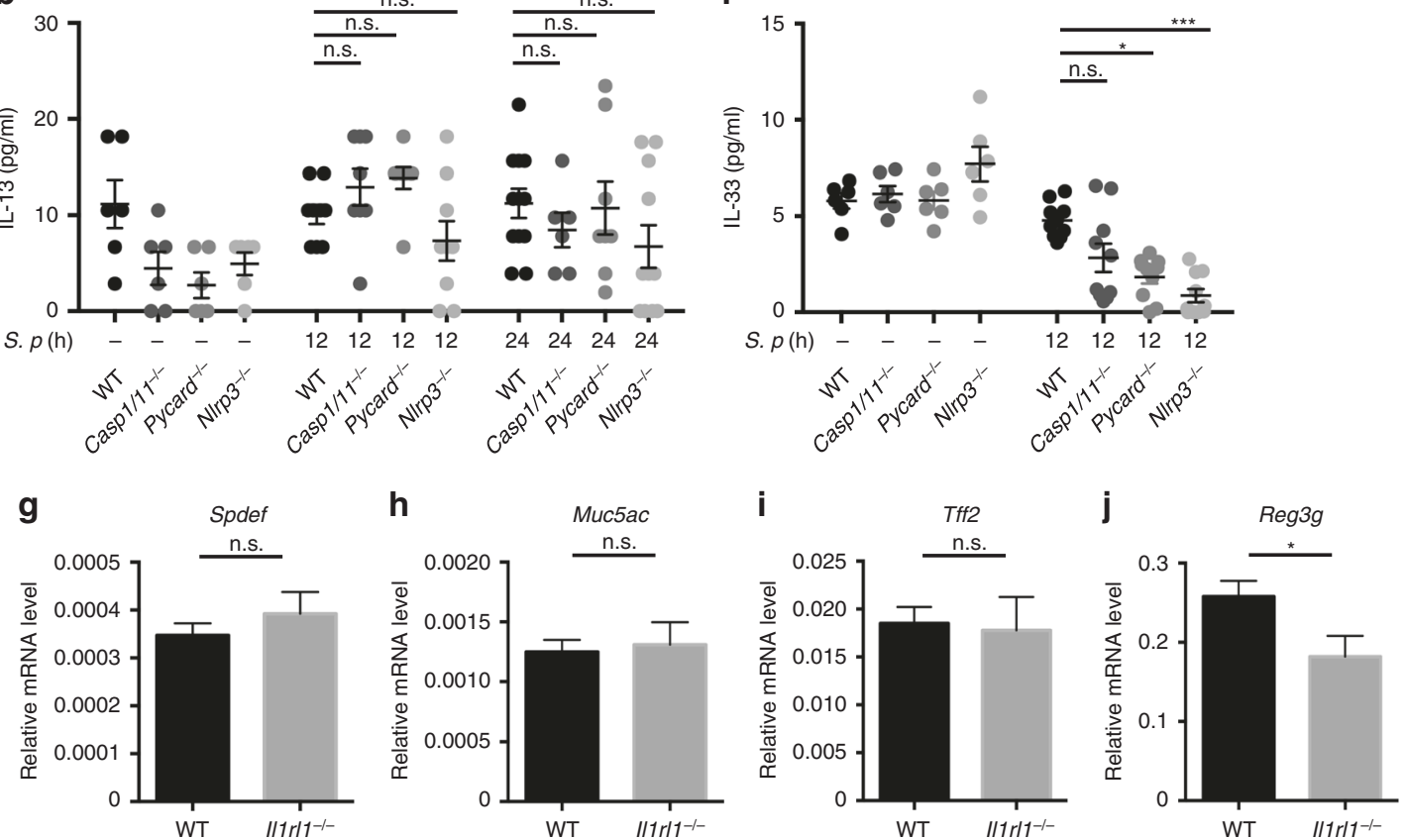

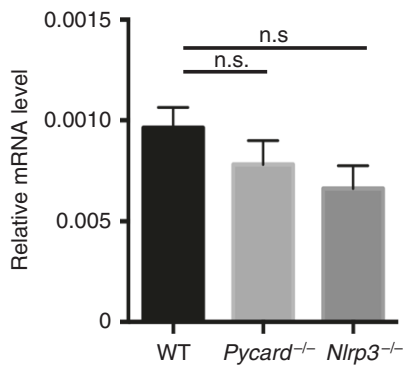

WT Pycard ${ }^{-/-}$Nlrp3 $^{-/}$

Fig. 6 Expression of type 2 cytokines during pneumococcal pneumonia. a, c-e Quantitative RT-PCR for assessing the levels of II13 (a), II25 (c), IL33 (d), and Tslp (e) mRNA relative to Actb mRNA in the lung of the indicated mice infected for $24 \mathrm{~h}$ with $\mathrm{S}$. pneumoniae ( $n=6$ ). b, $\mathbf{f}$ Quantification of IL-13 (b) and IL-33 (f) by ELISA in BALF of the indicated mice infected intranasally for $12 \mathrm{~h}$ or $24 \mathrm{~h}$ or not with S. pneumoniae $(n=6-12)$. g-j Quantitative RT-PCR for assessing the levels of Spdef (g), Muc5ac (h), Tff2 (i), and Reg3g (j) mRNA relative to Actb mRNA in the lung of the indicated mice infected for $24 \mathrm{~h}$ with S. pneumoniae $(n=6)$. $\mathbf{a}-\mathbf{j}$ Data are presented as the mean and SEM. ${ }^{*} p<0.05$; ${ }^{* *} p<0.01$; ${ }^{* *} p<0.001$; n.s. not significant by the Kruskal-Wallis test followed by Dunn's test (a-f) or by the Mann-Whitney $U$ test $(\mathbf{g}-\mathbf{j})$

important roles in innate immune defense against Gram-positive and Gram-negative bacteria, respectively. ${ }^{25,27,42-45}$ Considering that these ASC- and NLRP3-dependent mucosal defense factors (TFF2, REG3y, and BPIFA1) as a whole can provide protection against a broad range of microbial pathogens, it is worth examining if NLRP3 and ASC also maintain the expression of mucosal defense genes during infection by other microbial pathogens and in other mucosal tissues.

Although both ASC and NLRP3 were required for the optimal expression of Reg $3 g$, Tff2, and Bpifa1 during S. pneumoniae infection, the former, but not the latter, was required for it in uninfected mice (Fig. S3). One possible explanation for this is that ASC might maintain the expression of these genes dependently on NLRP3 upon pneumococcal pneumonia, but other receptors under normal conditions. This and other possibilities will be investigated in future studies.

S. pneumoniae infection reduced the expression of mucosal defense genes, such as Tff2 and Bpifa1, in the lung (Fig. S3). It has been suggested that IFN- $\gamma$ inhibits mucus production, blocks goblet cell development, and promotes goblet cell apoptosis. ${ }^{46} \mathrm{As}$ substantial levels of IFN- $\gamma$ were produced in response to $S$. pneumoniae infection in all groups tested, it is possible that mucus-producing goblet cells were decreased by IFN- $\gamma$ during pneumococcal pneumonia, resulting in the reduction in the expression of mucosal defense genes. By contrast, SPDEF mediates differentiation of goblet cells and plays a critical role in the expression of mucosal defense genes, including Reg3g, Tff2, Muc5b, and $I t \ln 1^{34-36}$ (GSE44101). Thus, we speculate that STAT6 activation, which is augmented by NLRP3 and ASC, maintains goblet cell differentiation and mucus production through the expression of SPDEF, thereby counteracting the effect of IFN- $\gamma$. The mechanism by which STAT6 was activated in airway epithelium during $S$. pneumoniae infection remains unclear. Low levels of IL-13 mRNA and protein were detected in S. pneumoniaeinfected mice (Fig. 6), implying that unlike allergens, this bacterium only weakly stimulates type 2 cytokine production, leading to modest STAT6 activation. However, further experiments are required to elucidate the mechanism linking $S$. pneumoniae infection to STAT6 activation.

In conclusion, we found that during pneumococcal pneumonia, ASC and NLRP3 maintain the expression of multiple mucosal defense genes in the airway mucosa, possibly by sustaining STAT6 
a
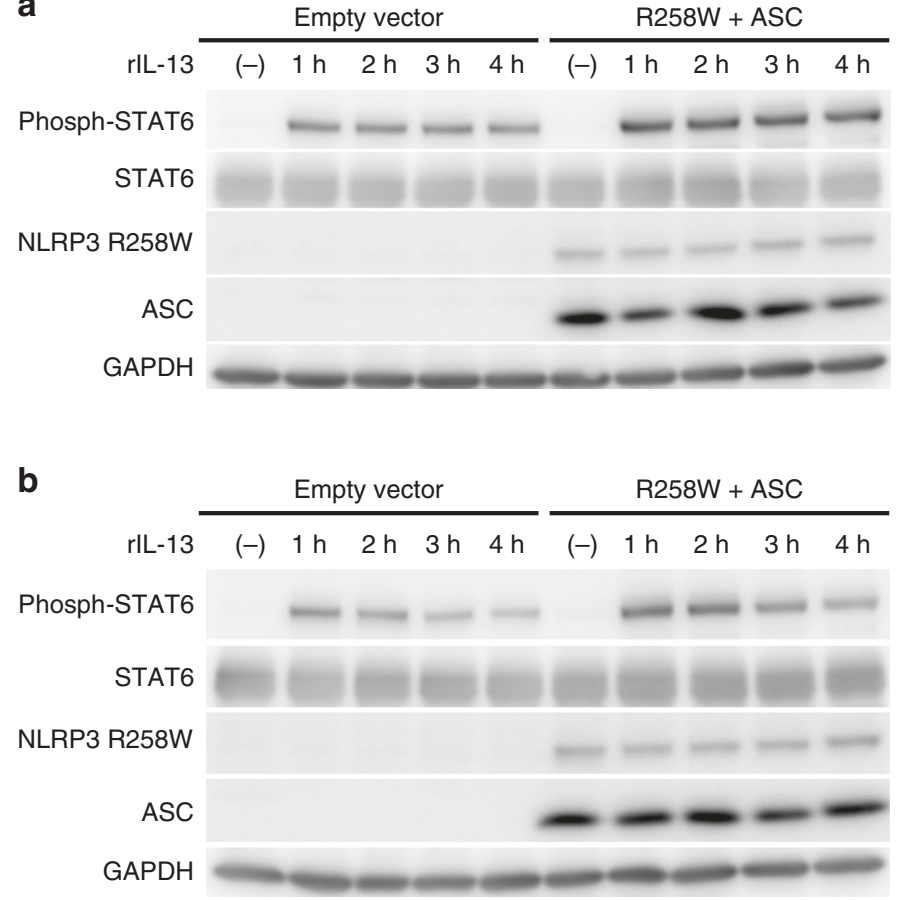
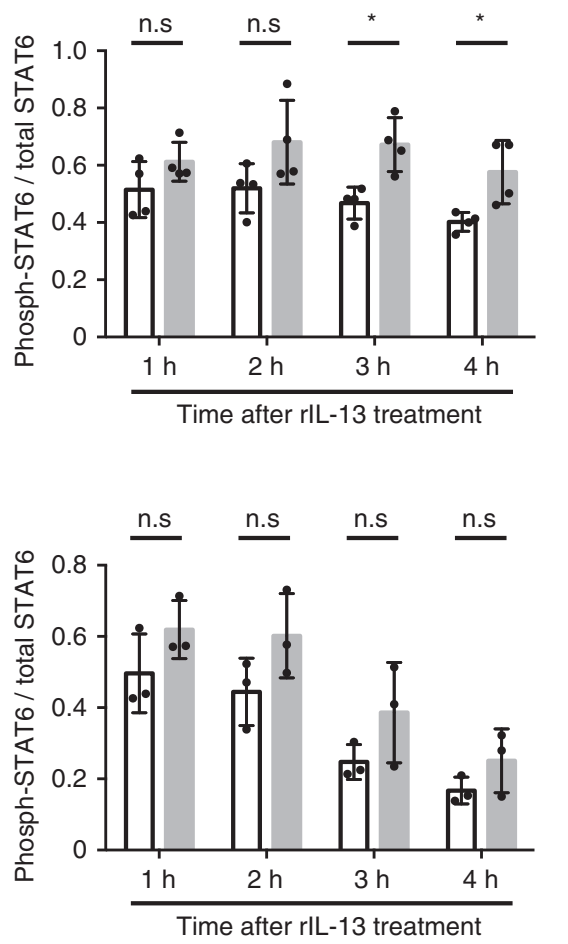

Empty vector

$\mathrm{R} 258 \mathrm{~W}+\mathrm{ASC}$
Empty vector $\mathrm{R} 258 \mathrm{~W}+\mathrm{ASC}$
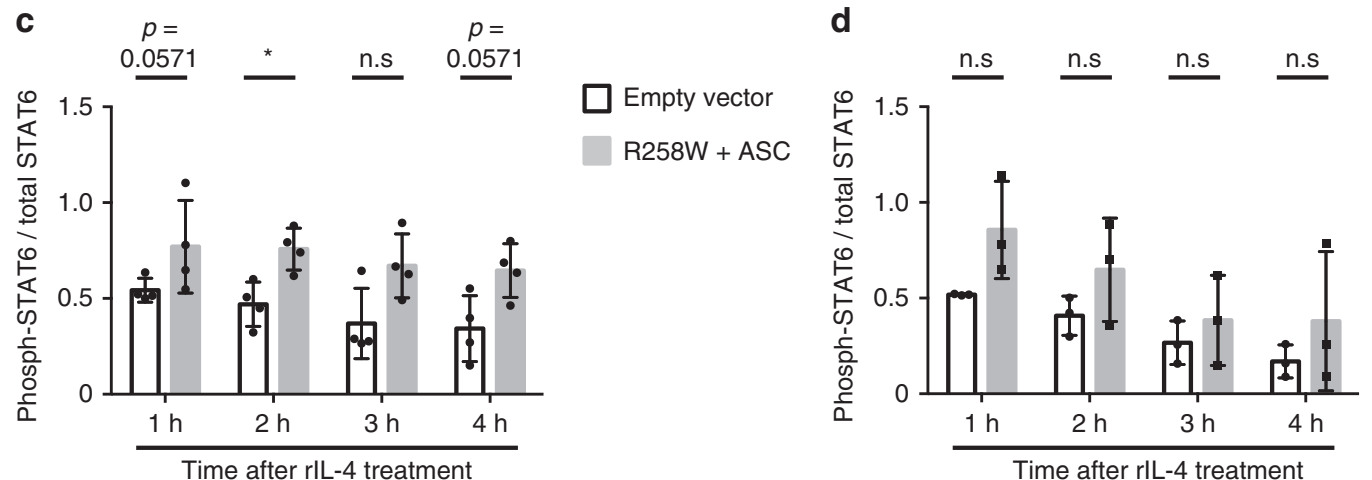

Fig. 7 ASC and NLRP3 sustain STAT6 activation. a, b Western blot analysis of phospho-STAT6 in HEK293 cells co-transfected with a STAT6 expression vector as well as with expression vectors for NLRP3 R258W plus ASC or an empty vector. The cells were treated with rlL-13 (1 ng/ $\mathrm{ml}$ ) for the indicated times (a). The cells were treated with rlL-13 $(1 \mathrm{ng} / \mathrm{ml})$ for $1 \mathrm{~h}$, washed 2 times with culture medium to remove IL-13, and then further incubated for $1-3 \mathrm{~h}$ (b). c, d HEK293 cells transfected as in A, B were treated with rlL-4 (1 ng/ml) as in A (c) or B (d), and phosphoSTAT6 in cell lysates was analyzed by Western blotting. Right panels $\mathbf{a}, \mathbf{b}$ and $\mathbf{c}$, $\mathbf{d}$ show the relative intensity of bands for phosph-STAT6 normalized to the intensity of bands for total STAT6 in the same samples. Data are presented as the mean and SD, and $p$ values are based on Mann-Whitney $U$ test $(\mathbf{a}-\mathbf{d}) .{ }^{*} p<0.05$; n.s. not significant. Data are from one representative of four (a) or three (b) independent experiments with similar results. $n=4(\mathbf{a}, \mathbf{c})$ or $3(\mathbf{b}, \mathbf{d})$

activation in an inflammasome-independent manner. Our results demonstrate a novel caspase-1-independent role for inflammasome proteins, which may help to control microbial infections.

\section{MATERIALS AND METHODS}

\section{Reagents}

Anti-caspase-1 Ab (sc-514) and anti-ASC Ab (sc-22514-R) were purchased from Santa Cruz Biotechnology (Santa Cruz, CA); antiNLRP3 mAb (Cryo-2) from Enzo Life Sciences (Farmingdale, NY); anti- $\beta$-actin mAb from Sigma-Aldrich (St. Louis, MO); anti-phoshoJNK Ab (4668), anti-phosho-ERK Ab (9101), and anti-phosho-STAT3 $\mathrm{Ab}$ (9145) from Cell Signaling Technology (Danvers, MA); anti-phosho-STAT6 Ab (ab28829) from Abcam (Cambridge, MA); anti-Flag Ab from Rockland Immunochemicals (Gilbertsville, PA); anti-mouse CD16/32 mAb (101320), PE-labeled anti-mouse F4/80 $\mathrm{mAb}$ (123109), and FITC-labeled anti-Gr-1 mAb (108405) from Biolegend (San Diego, CA); ELISA kits for mouse IL-1 $\beta$, IL-6, IL-10, IL-13, IL-17A, IL-33, and TNFa from eBioscience; ELISA kits for mouse TFF2, BPIFA1, intelectin-1, and mucin 5b from Cloud-Clone Corp. (Katy, TX); IL-18 ELISA kit from Medical \& Biological Laboratories (Nagoya, Japan); anti-IFN- $\gamma$ Abs and recombinant IFN- $\gamma$ from Thermo Scientific (Waltham, MA); recombinant hIL-4 and recombinant hIL-13 from PeproTech (Rocky Hill, NJ).

Mice

Pycard ${ }^{-1-}$ mice generated by Prof. Shun-ichiro Taniguchi (Shinshu University), Casp $1^{-1-}$ Casp $11^{129 \mathrm{mt} / 129 \mathrm{mt}}$ mice generated by Dr. Keisuke Kuida (Millennium Pharmaceuticals), N/rp3 $3^{-/-}$mice generated by Prof. Jürg Tschopp (University of Lausanne), and 
$\| 1 r_{1} 1^{-1-}$ mice generated by Prof. Shizuo Akira (Osaka University) were kindly gifted. The mutant and WT mice were on a C57BL/6 background. Mice were maintained in specific-pathogen-free conditions and used at 8-10 weeks of age. All of the experimental procedures performed on mice were approved by the Animal Ethics and Research Committee of Kyoto University Graduate School of Medicine, Kyoto, Japan (Med Kyo 13011), and by the Animal Ethics and Research Committee of Southwest University, Chongqing, China (Nos. 11-1025).

\section{Bacterial strain}

S. pneumoniae D39 (serotype 2) was purchased from the National Collection of Type Cultures (NCTC 7466; Central Public Health Laboratory, London, UK). Pneumococci were grown overnight on blood agar plates at $37^{\circ} \mathrm{C}$ and $5 \% \mathrm{CO}_{2}$. Colonies were inoculated into Todd-Hewitt broth (BD Biosciences, San Jose, CA) supplemented with $0.5 \%$ yeast extract, grown until mid-logarithmic phase (optical density at $600 \mathrm{~nm}$ [OD600] $=0.5$ ), and centrifuged at $6000 \times g$ for $15 \mathrm{~min}$. The bacterial pellet was suspended in phosphate-buffered saline (PBS) and stocked at $-80^{\circ} \mathrm{C}$. The concentration was determined by colony counting on blood agar plates.

In vivo infection experiments

Mice were anesthetized with pentobarbitone (Nacalai tesque, Kyoto, Japan) and infected intranasally with S. pneumoniae in $30 \mu \mathrm{l}$ PBS. Lungs collected from the infected mice were homogenized in PBS, serially diluted, and plated onto blood agar. CFUs were counted after overnight culture. BALF samples were collected in 1 $\mathrm{ml}$ PBS. The collected BALF samples were first centrifuged at $200 \times g$ for $3 \mathrm{~min}$ at $4^{\circ} \mathrm{C}$, and the supernatants were transferred to new tubes for cytokine detection. For intranasal transfection, 10 $\mu \mathrm{g}$ of each plasmid dissolved in $20 \mu \mathrm{l} \mathrm{TE}$ was mixed with $10 \mu \mathrm{l}$ of Transfectin Lipid Reagent (Bio-Rad Laboratories, Hercules, CA), incubated for $15 \mathrm{~min}$ at room temperature, and inoculated into mice as above.

Flow cytometric analysis

The pellets of BALF were washed and the total cell number was counted. Totally, $10^{6}$ cells from each sample were incubated with anti-mouse CD16/32 mAb for $30 \mathrm{~min}$ at $4{ }^{\circ} \mathrm{C}$. Then the cells were stained with PE-labeled anti-mouse F4/80 mAb and FITC-labeled anti-Gr-1 $\mathrm{mAb}$ for $30 \mathrm{~min}$ at $4{ }^{\circ} \mathrm{C}$ and analyzed on a FACScalibur flow cytometer (BD Biosciences). The number of $\mathrm{F} 4 / 80^{+}$macrophages and $\mathrm{F} 4 / 80^{-} \mathrm{Gr}-1^{+}$neutrophils were calculated.

Assay of neutrophil serine protease activity

BALF was diluted 10 times with NSP assay buffer $(50 \mathrm{mM}$ Tris- $\mathrm{HCl}$ pH7.4, $150 \mathrm{mM} \mathrm{NaCl}$ ) and incubated with $1 \mathrm{mM} \mathrm{N}$-Methoxysuccinyl-Ala-Ala-Pro-Val p-nitroanilide (MeOSuc-AAPV-pNA; SigmaAldrich) for $24 \mathrm{~h}$ at $37^{\circ} \mathrm{C}$, and the OD at $405 \mathrm{~nm}$ was measured with a plate reader.

Histopathology

The lungs were perfused with $10 \mathrm{ml}$ PBS, fixed with $10 \%$ formalin, and paraffin-embedded. Slides were stained with hematoxylin/eosin or with anti-phospho-STAT3 Ab or antiphospho-STAT6 Ab.

\section{Microarray}

Total RNAs were extracted from whole lungs using TRIzol Reagent (Thermo Scientific) followed by cleanup using NucleoSpin RNA Clean-up (MACHEREY-NAGEL, Düren, Germany). Microarray analysis was performed by using SurePrint G3 Mouse GE $8 \times 60 \mathrm{~K}$ Microarray (Agilent, Santa Clara, CA) at the Takara Bio Corporation (Shiga, Japan). Cy3-labeled cRNAs were synthesized using Low Input Quick Amp Labeling Kit One-Color (Agilent). Hybridization and washing were done by using Gene Expression Hybridization
Kit (Agilent) and Gene Expression Wash Buffers Pack (Agilent), respectively. Slides were scanned on an Agilent DNA Microarray Scanner G2565CA, and data were obtained using Agilent Feature Extraction software. Microarray data have been submitted to GEO (NCBI) under the GSE accession number GSE56653.

\section{Quantitative RT-PCR}

Total RNAs were isolated from lungs and small intestines as described above. The RNAs were reverse-transcribed using SuperScript VILO cDNA Synthesis Kit (Thermo Scientific). Quantitative real-time RT-PCR was carried out using primers listed in Table S3 (Primer No. 1-34), EXPRESS SYBR GreenER qPCR Supermix with Premixed ROX (Thermo Scientific), and the Applied Biosystems PRISM 7000 (Thermo Scientific), according to the manufacturer's instructions. Relative mRNA level was calculated by the $\Delta C T$ method with Actb mRNA for normalization.

\section{Western blotting analysis}

Proteins were extracted from whole lungs using TRIzol Reagent. The protein samples ( $1 \mu \mathrm{g}$ per sample) were subjected to sodium dodecyl sulphate (SDS)-polyacrylamide gel electrophoresis and subsequently transferred to polyvinylidene difluoride membranes (Merck Millipore, Billerica, MA). The membranes were immunoblotted with antibodies indicated in each figure.

\section{Plasmids}

The full-length SPDEF CDNA was amplified by PCR using primers Nos. 43 and 44 (Table S3) and then inserted into pFLAG-CMV2 vector (Sigma-Aldrich). The pFLAG expression vectors for TFF2, BPIFA1, BPIFB1, and intelectin-1 were constructed by using primers Nos. 35-42 (Table S3) and the pFLAG-CMV1 vector (Sigma-Aldrich). The expression vectors for ASC and NLRP3_R258W were constructed in our previous study. ${ }^{47}$ Luciferase reporter vector for the Reg $3 g$ gene promoter was constructed by using primers Nos. 45 and 46 (Table S3) and the pGL3-Basic vector (Promega, Madison, WI). The reporter vector pGL3-Reg3g promoter contain -1501 to +310 of the Reg $3 g$ gene. The pRLSV40 vector was purchased from Promega. pCMV-STAT6-IRES-Neo was a gift from Axel Nohturfft (Addgene plasmid \# 35482).

Protein binding assay

HEK293 cells obtained from the American Type Culture Collection were transiently transfected with $1 \mu \mathrm{g}$ of each expression vector using Transfectin Lipid Reagent (Bio-Rad Laboratories, Hercules, CA). Culture medium was replaced with Opti-MEM (Thermo Scientific) supplemented with $1 \%$ fetal calf serum 1 day after transfection, and the cells were incubated for an additional $48 \mathrm{~h}$. Culture supernatants were then collected and used for protein binding assay. Pneumococci were diluted in assay buffer (Tris buffered saline containing $2 \mathrm{mM} \mathrm{CaCl}_{2}$ ) and incubated at $4{ }^{\circ} \mathrm{C}$ for $2 \mathrm{~h}$ after the addition of $1 / 5$ volume of the HEK293 culture supernatants. Bacterial cells were washed twice with assay buffer, and S. pneumoniae-bound proteins were eluted in SDS-sample buffer. The samples were subjected to Western blot analysis with an anti-Flag $\mathrm{Ab}$.

\section{Luciferase assay}

HEK293 cells grown on 24-well plates were co-transfected with pRL-SV40 (50 ng) and either of pGL3-Basic, pGL3-Tff2 promoter or pGL3-Reg3g promoter $(500 \mathrm{ng})$. The cells were also transfected with pFLAG-CMV2 (300 ng), pFLAG-SPDEF (300 ng), or pFLAG-ASC $(150 \mathrm{ng})$ plus pFLAG-NLRP3_R258W $(150 \mathrm{ng})$. The cells were washed and lysed in $100 \mu$ l of Passive Lysis Buffer (Promega) 48 $\mathrm{h}$ after transfection. Firefly luciferase activity and Renilla luciferase activity in the lysates were measured using the Dual-Luciferase Reporter Assay System (Promega) and Wallac ARVO SX Multilabel Counter (Perkin Elmer, Waltham, MA). pGL3 Firefly luciferase activity was normalized to $\mathrm{pRL}$ Renilla luciferase activity. 


\section{Statistical analysis}

For comparisons between two groups, Mann-Whitney $U$ test was used. Multigroup comparisons were made by Kruskal-Wallis test followed by Dunn's test. Survival differences were analyzed by the log-rank test. For all statistical analyses, GraphPad Prism 6 software was used.

\section{ACKNOWLEDGEMENTS}

We thank Prof. Jürg Tschopp (University of Lausanne) and the Institute for Arthritis Research for providing N/rp3 ${ }^{-1-}$ mice; Prof. Shun-ichiro Taniguchi (Shinshu University) for Pycard ${ }^{-1-}$ mice; Dr. Keisuke Kuida (Millennium Pharmaceuticals) for Casp 1/11 $1^{-1-}$ mice; Prof. Shizuo Akira (Osaka University) for $\| 11 r 11^{-1-}$ mice; the center for anatomical and pathological researches of Kyoto University for histological support; Ms. Shoko Hosojima and Ms. Nana Iwami for technical assistance. This work was supported by the Japan Society for the Promotion of Science (JSPS KAKENHI Grant Numbers 26460523, 18K07106, 15K15078, 26110002, and 24590522), the Ministry of Health, Labor and Welfare of Japan, the National Key Research and Development Program of China (2018YFD0500500) and National Natural Science Foundation of China (31400762).

\section{AUTHOR CONTRIBUTIONS}

R.F. performed most of the experiments. R.U. performed the experiments with ST2deficient mice. S.S. helped with the microarray analysis. H.H. helped with the bone marrow chimera experiments. H.T. provided materials and reviewed the paper. M.M., I.K., and T.S. discussed the experimental results and reviewed the paper. K.T. supervised the study, designed the experiments, wrote the paper, and performed experiments.

\section{ADDITIONAL INFORMATION}

The online version of this article (https://doi.org/10.1038/s41385-019-0181-1) contains supplementary material, which is available to authorized users.

Competing interests: The authors declare no competing interests.

Publisher's note: Springer Nature remains neutral with regard to jurisdictional claims in published maps and institutional affiliations.

\section{REFERENCES}

1. Hallstrand, T. S. et al. Airway epithelial regulation of pulmonary immune homeostasis and inflammation. Clin. Immunol. 151, 1-15 (2014).

2. Tuomanen, E. I., Austrian, R. \& Masure, H. R. Pathogenesis of pneumococcal infection. N. Engl. J. Med. 332, 1280-1284 (1995).

3. Kadioglu, A., Weiser, J. N., Paton, J. C. \& Andrew, P. W. The role of Streptococcus pneumoniae virulence factors in host respiratory colonization and disease. Nat. Rev. Microbiol. 6, 288-301 (2008).

4. Marriott, H. M., Mitchell, T. J. \& Dockrell, D. H. Pneumolysin: a double-edged sword during the host-pathogen interaction. Curr. Mol. Med. 8, 497-509 (2008)

5. Kumar, H., Kawai, T. \& Akira, S. Pathogen recognition by the innate immune system. Int. Rev. Immunol. 30, 16-34 (2011).

6. Tsuchiya, K. \& Hara, H. The inflammasome and its regulation. Crit. Rev. Immunol. 34, 41-80 (2014).

7. Latz, E. The inflammasomes: mechanisms of activation and function. Curr. Opin. Immunol. 22, 28-33 (2010).

8. Koppe, U., Suttorp, N. \& Opitz, B. Recognition of Streptococcus pneumoniae by the innate immune system. Cell Microbiol. 14, 460-466 (2012).

9. Fang, R. et al. Critical roles of ASC inflammasomes in caspase-1 activation and host innate resistance to Streptococcus pneumoniae infection. J. Immunol. 187, 4890-4899 (2011).

10. McNeela, E. A. et al. Pneumolysin activates the NLRP3 inflammasome and promotes proinflammatory cytokines independently of TLR4. PLoS Pathog. 6, e1001191 (2010).

11. Shoma, S. et al. Critical involvement of pneumolysin in production of interleukin1alpha and caspase-1-dependent cytokines in infection with Streptococcus pneumoniae in vitro: a novel function of pneumolysin in caspase-1 activation. Infect. Immun. 76, 1547-1557 (2008).

12. Witzenrath, M. et al. The NLRP3 inflammasome is differentially activated by pneumolysin variants and contributes to host defense in pneumococcal pneumonia. J. Immunol. 187, 434-440 (2011).
13. Van, M. L. et al. ASC and NLRP3 impair host defense during lethal pneumonia caused by serotype 3 Streptococcus pneumoniae in mice. Eur. J. Immunol. 48, 66-79 (2018).

14. Kafka, D. et al. Contribution of IL-1 to resistance to Streptococcus pneumoniae infection. Int. Immunol. 20, 1139-1146 (2008).

15. Lauw, F. N. et al. IL-18 improves the early antimicrobial host response to pneumococcal pneumonia. J. Immunol. 168, 372-378 (2002).

16. Zwijnenburg, P. J., van der Poll, T., Florquin, S., Roord, J. J. \& Van Furth, A. M. IL-1 receptor type 1 gene-deficient mice demonstrate an impaired host defense against pneumococcal meningitis. J. Immunol. 170, 4724-4730 (2003).

17. Cash, H. L., Whitham, C. V., Behrendt, C. L. \& Hooper, L. V. Symbiotic bacteria direct expression of an intestinal bactericidal lectin. Science 313, 1126-1130 (2006).

18. Roy, M. G. et al. Muc5b is required for airway defence. Nature 505, 412-416 (2014).

19. Shah, A. A. et al. Increased susceptibility to Yersinia enterocolitica Infection of Tff2 deficient mice. Cell. Physiol. Biochem. 30, 853-862 (2012).

20. Shin, O. S. et al. LPLUNC1 modulates innate immune responses to Vibrio cholerae. J. Infect. Dis. 204, 1349-1357 (2011).

21. Taupin, D. \& Podolsky, D. K. Trefoil factors: initiators of mucosal healing. Nat. Rev. Mol. Cell Biol. 4, 721-732 (2003).

22. Tsuji, S. et al. Capture of heat-killed Mycobacterium bovis bacillus Calmette-Guerin by intelectin-1 deposited on cell surfaces. Glycobiology 19, 518-526 (2009).

23. Van Itallie, C. M. \& Anderson, J. M. Claudin interactions in and out of the tight junction. Tissue Barriers 1, e25247 (2013).

24. Zariwala, M. A., Knowles, M. R. \& Omran, H. Genetic defects in ciliary structure and function. Annu. Rev. Physiol. 69, 423-450 (2007).

25. $\mathrm{Di}, \mathrm{Y}$. P. Functional roles of SPLUNC1 in the innate immune response against Gram-negative bacteria. Biochem. Soc. Trans. 39, 1051-1055 (2011).

26. Lim, J. H. et al. Tumor suppressor CYLD regulates acute lung injury in lethal Streptococcus pneumoniae infections. Immunity 27, 349-360 (2007).

27. Choi, S. M. et al. Innate Stat3-mediated induction of the antimicrobial protein Reg3gamma is required for host defense against MRSA pneumonia. J. Exp. Med. 210, 551-561 (2013).

28. Wills-Karp, M. et al. Trefoil factor 2 rapidly induces interleukin 33 to promote type 2 immunity during allergic asthma and hookworm infection. J. Exp. Med. 209, 607-622 (2012).

29. Xu, Y., Ikegami, M., Wang, Y., Matsuzaki, Y. \& Whitsett, J. A. Gene expression and biological processes influenced by deletion of Stat3 in pulmonary type II epithelial cells. BMC Genomics 8, 455 (2007).

30. Tu S. P., et al. p53 inhibition of AP1-dependent TFF2 expression induces apoptosis and inhibits cell migration in gastric cancer cells. Am J Physiol Gastrointest Liver Physiol. 297, 385-396 (2009).

31. Thaikoottathil, J. \& Chu, H. W. MAPK/AP-1 activation mediates TLR2 agonistinduced SPLUNC1 expression in human lung epithelial cells. Mol. Immunol. 49, 415-422 (2011)

32. Hasegawa, M. et al. Mechanism and repertoire of ASC-mediated gene expression. J. Immunol. 182, 7655-7662 (2009).

33. Maeda, Y. et al. Airway epithelial transcription factor NK2 homeobox 1 inhibits mucous cell metaplasia and Th2 inflammation. Am. J. Respir. Crit. Care. Med. 184, 421-429 (2011).

34. Chen, G. et al. SPDEF is required for mouse pulmonary goblet cell differentiation and regulates a network of genes associated with mucus production. J. Clin. Invest. 119, 2914-2924 (2009).

35. Horst, D. et al. Requirement of the epithelium-specific Ets transcription factor Spdef for mucous gland cell function in the gastric antrum. J. Biol. Chem. 285, 35047-35055 (2010).

36. Marko, C. K. et al. Spdef null mice lack conjunctival goblet cells and provide a model of dry eye. Am. J. Pathol. 183, 35-48 (2013).

37. Park, K. S. et al. SPDEF regulates goblet cell hyperplasia in the airway epithelium. J. Clin. Invest. 117, 978-988 (2007).

38. Erle, D. J. \& Sheppard, D. The cell biology of asthma. J. Cell Biol. 205, 621-631 (2014).

39. Schmitz, J. et al. IL-33, an interleukin-1-like cytokine that signals via the IL-1 receptor-related protein ST2 and induces T helper type 2-associated cytokines. Immunity 23, 479-490 (2005).

40. Hebenstreit, D., Luft, P., Schmiedlechner, A., Duschl, A. \& Horejs-Hoeck, J. SOCS-1 and SOCS-3 inhibit IL-4 and IL-13 induced activation of Eotaxin-3/CCL26 gene expression in HEK293 cells. Mol. Immunol. 42, 295-303 (2005).

41. Kostadinova, E. et al. NLRP3 protects alveolar barrier integrity by an inflammasome-independent increase of epithelial cell adherence. Sci. Rep. 6, 30943 (2016).

42. Brandl, K., Plitas, G., Schnabl, B., DeMatteo, R. P. \& Pamer, E. G. MyD88-mediated signals induce the bactericidal lectin Reglll gamma and protect mice against intestinal Listeria monocytogenes infection. J. Exp. Med. 204, 1891-1900 (2007). 
ASC and NLRP3 maintain innate immune homeostasis in the airway through an... $\mathrm{R}$ Fang et al.

43. Liu, Y. et al. SPLUNC1/BPIFA1 contributes to pulmonary host defense against Klebsiella pneumoniae respiratory infection. Am. J. Pathol. 182, 1519-1531 (2013).

44. Liu, Y. et al. Increased susceptibility to pulmonary Pseudomonas infection in Splunc1 knockout mice. J. Immunol. 191, 4259-4268 (2013).

45. Vaishnava, S. et al. The antibacterial lectin Reglllgamma promotes the spatial segregation of microbiota and host in the intestine. Science 334, 255-258 (2011).

46. Mitchell, C., Provost, K., Niu, N., Homer, R. \& Cohn, L. IFN-gamma acts on the airway epithelium to inhibit local and systemic pathology in allergic airway disease. J. Immunol. 187, 3815-3820 (2011).

47. Hara, H. et al. Phosphorylation of the adaptor ASC acts as a molecular switch that controls the formation of speck-like aggregates and inflammasome activity. Nat. Immunol. 14, 1247-1255 (2013).
Open Access This article is licensed under a Creative Commons Attribution 4.0 International License, which permits use, sharing, adaptation, distribution and reproduction in any medium or format, as long as you give appropriate credit to the original author(s) and the source, provide a link to the Creative Commons license, and indicate if changes were made. The images or other third party material in this article are included in the article's Creative Commons license, unless indicated otherwise in a credit line to the material. If material is not included in the article's Creative Commons license and your intended use is not permitted by statutory regulation or exceeds the permitted use, you will need to obtain permission directly from the copyright holder. To view a copy of this license, visit http://creativecommons. org/licenses/by/4.0/.

(c) The Author(s) 2019 